\title{
Conservative Surgery for Ovarian Cancer
}

\author{
AMITA MAHESHWARI
}

The term conservative surgery for ovarian cancer indicates a surgical procedure that allows the removal of the ovarian tumour together with adequate staging procedures, while preserving the patient's reproductive potential. Ideally fertility potential should be preserved without compromising cure rates. Conservative surgery has been shown to be onocologically safe in certain histological subtypes of ovarian cancers such as malignant ovarian germ cell tumours, granulaosa cell tumours, borderline ovarian tumour and early stage, low-intermediate grade, invasive epithelial ovarian cancer.

Malignant ovarian germ cell tumours (MOGCT) are rare but curable even in advanced stages of disease. Appropriate surgical treatment for patients where fertility needs to be preserved consists of staging laparotomy with unilateral salpingo-oophorectomy (USO). Conventionally, adjuvant chemotherapy is recommended in all cases except stage-IA dysgerminoma and stage IA grade-1 immature teratoma.

Borderline ovarian tumour (BOT) represents 10$20 \%$ of epithelial ovarian cancers. The mean age at diagnosis of BOT is 10 years younger than that of invasive EOC and $1 / 3^{\text {rd }}$ of BOTs are diagnosed in patients aged less than 40 years. Conservative surgery is the standard of care in these cases. Although such treatments increase the rate of recurrences (15-35\% depending on the type of surgery i. e. oophorectomy vs ovarian cystectomy) however this does not adversely impact survival as most recurrences are of borderline type and can easily be treated

Associate Professor of Gynecologic Oncology

Tata Memorial Hospital, Mumbai

E-mail: maheshwariamita@yahoo.com with surgery.

Nearly $15 \%$ of invasive epithelial ovarian cancer (EOC) occurs in women younger than 40 years. The role of conservative surgery in invasive EOC is less well defined. The prognosis for patients who develop a recurrence after fertilitysparing surgery remains poor. Therefore, initial selection of candidates for fertility-sparing surgery should be done carefully. The patient and the family should be extensively counseled. The patient should be aware of slightly increased risk for recurrence associated with conservative surgery. Furthermore, patient needs to be assessed for the realistic probabilities of achieving conception on the basis on their age, history, and infertility evaluation. Most authors agree that conservative surgery can be offered to patients with stage Ia and grade 1-2, non clear cell EOC. A comprehensive surgical staging is mandatory because occult extra-ovarian metastases can occur in a significant proportion of women with apparent early-stage disease. Opposite adnexal should be carefully evaluated however; random biopsies or bisection of normal looking ovary is not indicated.

The optimal interval between completion of cancer treatment and conception must be carefully determined by a multidisciplinary team including oncologists and obstetrician. The general recommendation is to wait for one to two years after completion of treatment before attempting conception. However, delaying conception for too long has the risk of premature ovarian failure in these patients with low oocyte reserve. The reproductive outcomes of fertility-sparing treatment are promising and majority of patients can expect spontaneous conception. However, $10-20 \%$ patients will fail 
to conceive naturally and will require assisted reproduction technology (ART) to achieve a pregnancy, especially in-vitro fertilization (IVF). Although some retrospective studies in the past have reported increased risk of EOC in patients undergoing ART, more recent literature does not support this observation and ART is believed to be oncologically safe. A normal pregnancy outcome can be expected in most cases although a few studies have reported slightly increased risk of congenital malformation and miscarriages in ovarian cancer patients treated with surgery and chemotherapy. The role of newer reproductive techniques e.g. ovarain tissue cryopreservation, oocyte cryopreservation, embryo cryopreservation needs further exploration in selected cases of ovarian cancer. The role of completion surgery i.e. removal of the uterus and adnexal at the completion of fertility is debatable. Completion of surgery can be reasonably deferred until menopause if the patient agrees to careful follow-up.

Management of young women with ovarian cancer should be individualized; the risk of conservative therapy should be balanced against the disadvantages of more radical treatment. Usually a multidisciplinary approach with close collaboration among gynecologic oncologist, obstetrician and perinatologist is required to have successful oncologic and obstetric outcomes. 